that statistically significant results should not be unthinkingly disparaged. However, his attractive Bayesian formulation is still based on a subjective assessment of the true probability, $t$ in his notation, that the alternative hypothesis is true.

Using his formula, the probability of a significant result being a true positive varies remarkably little under a wide range of plausible assumptions. For example, if $t=0.5, \alpha=0.01$, and the statistical power is 0.5 , then his formula yields 0.98 as this probability, while for a much lower statistical power $(0.1)$ this probability is still 0.91 . Only with $\alpha=0.05$ and a power of 0.1 or other comparably dismal assumptions - which, however, may apply in real cases - does the probability

\section{Health care costs}

To the editor - In your editorial "Rising health-care costs inevitable" (1, 975, 1995) it was said that "those of us who are neither politicians nor policy wonks" would be helped "if the arguments for cost savings were cast in human rather than macroeconomic terms." More than ten years ago I wrote on this subject (Health \& Social Service Journal 92, 1462; 1982) demonstrating that huge cost reductions could be obtained by amalgamating the training of junior hospital doctors and nurses into one work force, termed 'stem doctors'. I argued that the majority of medicine carried out worldwide could be performed by following relatively simple procedures and could be administered by medical staff trained for three or four years at most. This would result in more and cheaper health workers where they are needed most. I also argued that the spin off to this approach would be a greatly reduced use of high-technology medical facilities when their use was inappropriate. of a significant result being a true positive fall as low as 0.666. The Bayesian calculation thus agrees with the commonsense notion that it is best to execute and publish studies where the power is high and the significance level conservative.

A scientific finding must be replicated to be widely accepted. The statistical significance of a finding is an important guide, but not the only one, to practical decisions. An investigator must decide whether to pursue additional independent data or to design new research. A journal must decide whether to publish the finding and how much space to devote to it. Other investigators decide whether they wish to follow up on the finding. Granting agencies choose whether to fund further

This proposal met with little enthusiasm because of the vested interest of nurses and doctors; both groups wished to protect their status and traditions. Unless we do follow through with these or similar ideas, we will continue to duplicate and reduplicate a truly amazing amount of teaching, administrative, laboratory and clinical work, much of which could have been carried out closer to the ultimate starting point in medicine - the patient.

\section{A.D.B. CHANT}

Royal South Hants Hospital

Brintons Terrace

Southampton SO14 OYG, UK

To the editor - Your editorial on the escalating cost of health care (Nature Medicine $1,975 ; 1995)$ misses an important issue. While you express concern about the problem of health care in affluent nations, you have totally ignored the plight of patients in the other half of the world. In India, a land of 900 million people, more than 300 million live in poverty. research based on this finding. Such decisions take into account not only the level of statistical significance but also the potential clinical or scientific importance of the finding and its subjective "biological plausibility." These considerations may be more important than possible publication bias; indeed, extreme emphasis on possible publication bias could lead one to reject all studies with statistically significant findings as well as all those with no significant result.

MiCHAEL SWIFT

The Institute for the Genetic Analysis of Common Diseases

New York Medical College, 4 Skyline Drive

Hawthorne, New York 10532, USA

Even for the middle class, also numbering about 300 million, an echocardiogram means a week's salary, a coronary angiogram a year's salary and coronary bypass surgery means more than one's life's savings. The majority need to work for 30 months and spend all that they earn to buy an artificial valve. Affluent developed nations are vying for expanding the market for their health-care products to cater to the minority of the population in developing countries who can either buy the product or get support to buy it. The majority of the sick suffer unknown, unsung and unwept. Even funding for health care through banks like the Asian Development Bank burdens the end consumer with $27 \%$ interest, ultimately paid by the poor patient. There is virtually no social security. Insurance coverage is meagre. Do the economists have a solution?

\section{SOLOMON VICTOR}

The Heart Institute

Vijaya Health Centre

N.S.K. Salai, Vadapalani

Madras 600 026, India

\section{A cornucopia of drug discovery?}

To the editor - In their Commentary', Wurtman and Bettiker balance the current contention that reductive biological sciences techniques will write the book of revelation of all future drugs. "The slowing of treatment discovery, 1965-1995" is blamed in part on "misunderstanding of the treatment discovery process." This in turn probably reflects our adjusted teach- ings and textbooks that, for the most part, focus on reductive and theoretic schemes generated after the treatment discovery has already been made. Some examples, not widely known, from the antiasthma drug field may further underscore the value of exploratory in vivo approaches (including "clinical investigations and off-label drug studies") in treatment discovery processes.

Hyde Salter (1823-1870) advocated "simple reading of nature" and reacted strongly when "its place (was) supplied by an unquestioning inheritance and adoption of received notions." Salter discovered many aspects of asthma including the fact that violent emotions, causing the release of "a nervous derivative," could produce instantaneous antiasthma effects ${ }^{2}$. When adrenaline eventually became available, its first suc- 